Total number of pages: 25

Total number of tables: 0

Total number of figures: 11

Total number of references: 21

\title{
POWER ABSORBED DURING WHOLE-BODY FORE-AND-AFT VIBRATION: EFFECTS OF SITTING POSTURE, BACKREST, AND FOOTREST
}

\author{
Naser Nawayseh ${ }^{a}$ and Michael J. Griffin ${ }^{b}$ \\ aDepartment of Mechanical and Mechatronics Engineering, College of Engineering, \\ Dhofar University, PO Box 2509, Postal Code 211 Salalah, Sultanate of Oman
}

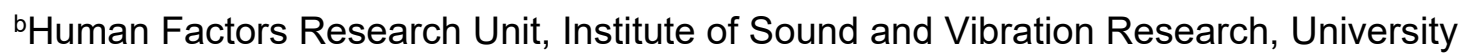
of Southampton, Southampton SO17 1BJ, UK

Correspondence address:

Dr Naser Nawayseh,

Department of Mechanical and Mechatronics Engineering,

College of Engineering,

Dhofar University,

PO Box 2509, Postal Code 211 Salalah,

Sultanate of Oman

e-mail: nnawayseh@yahoo.co.uk 


\begin{abstract}
Although the discomfort or injury associated with whole-body vibration cannot be predicted directly from the power absorbed during exposure to vibration, the absorbed power may contribute to understanding of the biodynamics involved in such responses. From measurements of force and acceleration at the seat, the feet, and the backrest, the power absorbed at these three locations was calculated for subjects sitting in four postures (feet hanging, maximum thigh contact, average thigh contact, and minimum thigh contact) both with and without a rigid vertical backrest while exposed to four magnitudes $\left(0.125,0.25,0.625\right.$, and $1.25 \mathrm{~ms}^{-2}$ r.m.s.) of random fore-and-aft vibration. The power absorbed by the body at the supporting seat surface when there was no backrest showed a peak around $1 \mathrm{~Hz}$ and another peak between 3 and $4 \mathrm{~Hz}$. Supporting the back with the backrest decreased the power absorbed at the seat at low frequencies but increased the power absorbed at high frequencies. Foot support influenced both the magnitude and the frequency of the peaks in the absorbed power spectra as well as the total absorbed power. The measurements of absorbed power are consistent with backrests being beneficial during exposure to low frequency fore-and-aft vibration but detrimental with high frequency fore-and-aft vibration.
\end{abstract}




\section{INTRODUCTION}

Current standards for the measurement, the evaluation, and the assessment of wholebody vibration are greatly influenced by understanding of the motions that cause vibration discomfort. This understanding has influenced methods for measuring, evaluating, and assessing vibration with respect to performance and health [1-3]. It seems reasonable to assume that the prediction of all human responses to vibration would be enhanced by greater understanding of biodynamic responses to vibration.

Biodynamic responses to whole-body vibration have mostly been reported in terms of the transmission of vibration from seat to head [4] or the apparent mass or mechanical impedance of the body [5-7]. The power absorbed in the body during whole-body vibration has also been reported [8-12]. Absorbed power has the advantage of allowing scalar summation: the overall power absorbed by the body can be found by adding the absorbed power in each direction of excitation and at all interfaces between the body and vibrating surfaces (e.g., at the seat, the back, and the feet). Another advantage sometimes claimed for absorbed power over apparent mass and transmissibility is that it includes duration in addition to the magnitude, frequency, and direction of vibration. Assuming the dependence on magnitude, frequency, direction, and duration implicit in absorbed power gives a good representation of how the body is affected by vibration, it has been suggested that the power absorbed by the body might be used to predict subjective responses to vibration (e.g. $[8,9]$ ). However, even if absorbed power has no direct applicability to the prediction of discomfort (or injury) it may contribute indirectly to understanding of the biodynamic responses that are involved in such responses to whole-body vibration.

Experimental studies of biodynamic responses to whole-body vibration have mostly investigated responses to vertical excitation. A few studies have reported the apparent mass [6], mechanical impedance [13], or transmissibility [14] to the head during horizontal excitation, but only three studies are known to have reported the power absorbed during horizontal excitation [9,15,16]. From experimental measurements of the mechanical impedance of subjects seated without armrests or backrests, Lee and Pradko [9] calculated the absorbed power during fore-and-aft and lateral excitation. It is not clear from the study whether a footrest was used. With males and females sitting on a plate (with no backrest and stationary feet), Lundström and Holmlund [15] investigated the power absorbed during exposure to fore-and-aft, lateral, and vertical excitation. The absorbed power varied with the direction of vibration excitation, and peaked at frequencies less than $2.5 \mathrm{~Hz}$ during horizontal excitation, compared to 4 to 6 
$\mathrm{Hz}$ during vertical excitation. The absorbed power was reported to depend on gender, leading to the suggestion that more restrictive risk assessment guidelines may be required for females [15]. Using fore-and-aft and lateral excitation and three backrest conditions (no back support, vertical backrest, and inclined backrest), Rakheja et al. [16] measured the absorbed power between the body and a seat, including the power absorbed between the back and a backrest. Their measures were obtained by mounting a seat with a backrest on a force measuring system and, in addition, measuring the fore-and-aft force at the backrest. They used the measurements to estimate the total absorbed power at the seat pan and the backrest and found that the backrest had a strong influence on both the power absorbed and how it depended on the frequency of fore-and-aft vibration.

Support for the feet and the position of the feet have been reported to affect the apparent mass measured at the seat surface during fore-and-aft excitation [6]. Foot support and foot position have also been found to affect both the apparent mass and the absorbed power at the seat during vertical excitation $[7,10]$. The effects of foot support and foot position on the power absorbed by the body during fore-and-aft excitation have not previously been reported.

When seated and exposed to fore-and-aft excitation, the manner in which contact with a backrest influences vibration discomfort depends on the frequency of vibration and the location of contact with the back [17-19]. In current standards, the effect of foreand-aft backrest vibration on discomfort is reflected in a simple frequency weighting, $W_{c}[2,3]$. The frequency-weighted vibration at the back is summed with the weighted vibration at other locations, so backrest vibration is always predicted to increase vibration discomfort. Similarly, vibration of the feet causes vibration discomfort in seated people [17,19] and standards include a frequency weighting for predicting vibration discomfort at the feet, $W_{\mathrm{b}}[2,3]$. The interpretation of studies of vibration discomfort at the back and the feet would be enhanced by an understanding of the frequency-dependence of the power absorbed at these locations, the extent to which the absorbed power differs between these locations, and how it depends on characteristics of a backrest (e.g. size and inclination) and a footrest (e.g. height). This will also contribute to understanding of whether the dependence of vibration discomfort on the frequency of vibration or the form of contact with vibration can be estimated from the power absorbed at the seat, the back, and the feet.

This paper reports the power absorbed by the human body from fore-and-aft vibration excitation applied at the seat, the back, and the feet. The main objective was to 
determine to what extent the power absorbed by the body during fore-and-aft excitation is affected by contact with a vibrating footrest and a vibrating backrest. It was hypothesised that the power absorbed from vibration at the seat would vary with posture, due to variations in the percentages of the body weight supported by the seat, the footrest, and the backrest. The data used in this paper are those from a study of the apparent masses of the body $[6,20]$ but reanalysed to calculate the absorbed power.

\section{APPARATUS, EXPERIMENTAL DESIGN AND ANALYSIS}

\subsection{Apparatus}

Subjects were exposed to random fore-and-aft vibration using an electro-hydraulic vibrator capable of producing a peak-to-peak displacement of 1 metre. A rigid seat was rigidly mounted on the platform of the vibrator in two conditions: without a backrest (data from Nawayseh and Griffin [6]) and with a vertical rigid backrest (data from Nawayseh and Griffin [20]). Both studies used an adjustable footrest (to give different foot heights) that moved horizontally in phase with the seat. Force signals from force plates (Kistler 9281 and Kistler Z 13053) were amplified by Kistler 5007 charge amplifiers so as to measure the forces at the seat, the backrest, and the footrest. Foreand-aft acceleration was measured at the centre of the force platforms using piezoresistive accelerometers (Entran EGCSY-240D-10). The signals from the accelerometers and the force transducers were digitised at 200 samples per second after passing through $67 \mathrm{~Hz}$ anti-aliasing filters.

\subsection{Experimental design}

The data from Nawayseh and Griffin [6] (i.e. without a backrest) were obtained with 12 male subjects having an average age of 28 years (range 21 to 38 years), weight 71.3 $\mathrm{kg}$ (range 56 to $87 \mathrm{~kg}$ ), and stature $1.72 \mathrm{~m}$ (range 1.63 to $1.83 \mathrm{~m}$ ). During exposure to fore-and-aft vibration, the fore-and-aft force and acceleration at the seat were measured. The same subjects were used by Nawayseh and Griffin [20] (i.e. with backrest) except for one subject, which changed the subject characteristics to average age 30.8 years (range 24 to 47 years), weight $76.1 \mathrm{~kg}$ (range 63 to $103 \mathrm{~kg}$ ), and stature $1.79 \mathrm{~m}$ (range 1.68 to $1.91 \mathrm{~m}$ ). During exposure to vibration, fore-and-aft accelerations at the seat and backrest as well as the fore-and-aft forces at the seat and backrest were measured simultaneously. The subjects in both studies were exposed to random fore-and-aft vibration with an approximately flat constant bandwidth acceleration power spectrum over the frequency range 0.25 to $20 \mathrm{~Hz}$ [6] or 0.25 to $10 \mathrm{~Hz}$ [20]. Additionally, a separate experiment was conducted to investigate the absorbed power at the feet during fore-and-aft whole-body vibration. Measurements of the fore-and-aft force and 
fore-and-aft acceleration at the feet of six subjects were recorded with $0.125,0.25$, and $0.625 \mathrm{~ms}^{-2}$ r.m.s. random vibration over the frequency range 0.25 to $10 \mathrm{~Hz}$.

Both studies employed the same exposure conditions: all 16 combinations of four vibration magnitudes $\left(0.125,0.25,0.625\right.$, and $1.25 \mathrm{~ms}^{-2}$ r.m.s.) and four sitting postures. The four sitting postures were achieved by changing the height of an adjustable footrest while keeping the upper body in an upright posture. The postures were: (i) 'feet hanging' with no foot support, (ii) feet supported with 'maximum thigh contact' (i.e. heels just in touch with the footrest), (iii) 'average thigh contact' (i.e. upper legs horizontal, lower legs vertical and supported on the footrest), and (iv) 'minimum thigh contact' (i.e. the footrest $160 \mathrm{~mm}$ above the position with 'average thigh contact' in position (iii)). When measuring forces at the feet, only the average thigh contact posture was used.

Each exposure to vibration lasted 60 seconds, and there was an interval of 2 to 3 minutes between exposures. It was not possible to measure the absorbed power when sitting in contact with all three interfaces (the seat, backrest, and feet) simultaneously due to equipment limitations. Although measurements at the seat and backrest were obtained simultaneously, measurements at the feet were obtained in a separate session. Although this study refers to four vibration magnitudes, the interpretation of the findings should recognise that over the frequency range 0.25 to $10 \mathrm{~Hz}$ the vibration magnitudes were lower when the frequency range of the excitation was 0.25 to $20 \mathrm{~Hz}$ [6] than when the frequency range was 0.25 to $10 \mathrm{~Hz}$ [20]. With the same r.m.s. magnitude of vibration over both bandwidths, the vibration magnitude over the range 0.25 to $10 \mathrm{~Hz}$ was greater (by a factor of approximately $\sqrt{2}$ ) when vibration was only presented over this narrower bandwidth, and the absorbed power was therefore also increased."

\subsection{Analysis}

The instantaneous power, $P$, transmitted to the human body during vibration can be calculated from the product of the force, $F$, and velocity, $v$, measured at the interface between the body and the vibrating surface. In this study, the velocity was obtained by integrating the measured acceleration time history. The power transmitted to the body can be calculated in the frequency domain from the cross-spectrum between the force and the velocity.

The real part of the transmitted power represents the power absorbed by the body:

$$
P_{\mathrm{Abs}}(f)=\operatorname{Re}\left\{G_{v F}(f)\right\}=\left|G_{v F}(f)\right| \cos \phi(f)
$$


where $P_{\text {Abs }}(f)$ is the absorbed power, $\operatorname{Re}\left\{G_{\mathrm{VF}}(f)\right\}$ is the real part of the cross-spectrum between the velocity and the force, $\left|G_{\mathrm{vF}}(f)\right|$ is the modulus of the cross-spectrum between the velocity and the force, $\phi$ is the phase of the cross-spectrum between the velocity and the force, and $f$ is the frequency. The absorbed power spectrum, $P_{\text {Abs }}(f)$, has units of $\mathrm{Nms}^{-1} / \mathrm{Hz}$. The imaginary part of the transmitted power represents the power that enters and leaves the body (i.e., there is energy exchange between the body and the vibrating surface during each cycle of motion).

The total power absorbed at each input interface was obtained by integrating the absorbed power spectra over the frequency range 0.78 to $10 \mathrm{~Hz}$. The unit of the total absorbed power is watts. The effects of using a footrest at different heights on the total absorbed power measured at the seat and at the backrest were examined through statistical analysis using SPSS (version 15.0). The effects of placing the feet on a footrest on the overall absorbed power (i.e. sum of total absorbed power at different interfaces) was also analysed statistically.

Non-parametric statistical techniques were employed because the data were not expected to be normally distributed: the Wilcoxon matched-pairs signed ranks test and the Spearman rank correlation. All statistical analysis to compare between the results of the first experiment and the results of the second experiment were performed using only the 11 subjects who took parts in both experiments.

\section{RESULTS}

\subsection{Effect of footrest on absorbed power}

In all four sitting postures, the absorbed power measured at the seat when there was no backrest was low at low frequencies but increased with increasing frequency to a peak around $1 \mathrm{~Hz}$ (Figure 1 and Figure 2). A second peak, with lower magnitude than the first peak, was evident between 3 and $4 \mathrm{~Hz}$ and clearer with the foot supported postures. With low vibration magnitudes ( 0.125 and $0.25 \mathrm{~ms}^{-2}$ r.m.s.), the principal peak in the median absorbed power tends to occur at a slightly higher frequency with the feet hanging than with the other three postures. There was no clear effect of the height of the foot support on the frequency at which maximum power was absorbed among the three postures with the feet supported (i.e. maximum thigh contact, average thigh contact, and minimum thigh contact postures). The peak median absorbed power was generally greater with the feet hanging than with the other three postures. The peak median absorbed power was greater with the minimum thigh contact posture than with the other feet supported postures and sometimes similar to, or even greater than, the 
feet hanging posture. Individual data exhibited similar trends to those shown by the median data.

\section{FIGURE 1 and FIGURE 2 ABOUT HERE}

As expected, the absorbed power at the seat increased with increasing magnitude of vibration (Figure 3). The power absorbed with $0.125 \mathrm{~ms}^{-2}$ r.m.s. was small and cannot be seen using the linear scale in Figure 2 but is evident on a logarithmic scale (Figure 4). Similarly, the power absorbed at the feet also increased with increasing magnitude of vibration (Figure 5).

\section{FIGURES 3 AND 4 AND 5 ABOUT HERE}

With no backrest and with the feet hanging, at all vibration magnitudes the total absorbed power measured at the seat was greater than with the other three postures $(p<0.006$, Wilcoxon). In the three postures where the feet were supported, there were no significant differences in the total absorbed power at the two lowest vibration magnitudes ( $p>0.05$, Wilcoxon). However, at $0.625 \mathrm{~ms}^{-2}$ and $1.25 \mathrm{~ms}^{-2}$ r.m.s., the total absorbed power with the minimum thigh contact posture was significantly greater than the total absorbed power with both the maximum thigh contact posture and the average thigh contact posture.

With the backrest and with the feet hanging, at all vibration magnitudes the total absorbed power measured on the seat was significantly greater than with the other three postures ( $p<0.05$, Wilcoxon), except the average thigh contact posture at 0.125 $\mathrm{ms}^{-2}$ r.m.s. ( $p>0.1$, Wilcoxon) and both the maximum thigh contact posture and the average thigh contact posture at $0.25 \mathrm{~ms}^{-2}$ r.m.s. ( $p>0.2$, Wilcoxon). With the feet supported, there were significant differences in the total absorbed power on the seat between the maximum thigh contact posture and the minimum thigh contact posture at $0.625 \mathrm{~ms}^{-2}$ r.m.s. and between the maximum thigh contact posture and both the average thigh contact posture and the minimum thigh contact posture at $1.25 \mathrm{~ms}^{-2}$ r.m.s.

Statistical analysis showed no significant differences between the total absorbed power measured at the backrest with the different postures at any vibration magnitude $(p>$ 0.053 , Wilcoxon), except at $1.25 \mathrm{~ms}^{-2}$ between the feet hanging posture and both the maximum thigh contact posture and the minimum thigh contact postures, between the maximum thigh contact posture and the minimum thigh contact posture, and between the average thigh contact posture and the minimum thigh contact posture $(p<0.034$, 
Wilcoxon). In these exceptional cases with the highest vibration magnitude, the total absorbed power increased with increasing height of the footrest.

\subsection{Effect of backrest on absorbed power}

Two peaks, one between 1 and $2 \mathrm{~Hz}$ and the other between 4 to $5 \mathrm{~Hz}$, were evident in the absorbed power measured on the seat with all postures when using a backrest (Figure 6). The absorbed power associated with the second peak was greater than that of the first peak, contrary to the absorbed power at the seat without a backrest (compare Figures 2 and 6).

\section{FIGURE 6 ABOUT HERE}

The power absorbed from the seat when sitting with the backrest depended on whether the feet were supported or not: with the feet hanging, the first peak was greater and clearer than with the other three postures with supported feet (Figure 6). The second peak tended to be smaller with the feet hanging than in the other three postures.

In all postures, as the vibration magnitude increased, the absorbed power increased and the frequency at which the absorbed power was greatest decreased (Figure 6, notice the different scales of the subplots). When sitting with a backrest, the power absorbed from vibration at the backrest was significantly greater than the power absorbed from vibration at the seat, and tended to occur over a wider range of frequencies (Figure 7). The total power absorbed at the backrest was found to be greater than the total power absorbed at the seat $(p<0.015$, Wilcoxon). With increasing magnitude of vibration, the power absorbed at the backrest increased and the frequency of the peak decreased (Figure 8).

\section{FIGURE 7 AND 8 ABOUT HERE}

Contact with the rigid flat vertical backrest tended to decrease the absorbed power at the seat at low frequencies but increase the absorbed power at the seat at high frequencies (Figure 9). This was also the case for the power absorbed at the feet (Figure 5). The low frequency power absorbed from vibration at the seat when sitting without a backrest was greater than the sum of the low frequency power absorbed at the seat and the backrest when sitting with a backrest (Figure 9). Figure 9 shows data for the minimum thigh contact posture at $1.25 \mathrm{~ms}^{-2}$ r.m.s. but the same trend was exhibited in all postures and at all vibration magnitudes (Figure 10). 
As explained in Section 2.2, it was not possible to measure the absorbed power simultaneously at all interfaces (i.e. seat, backrest, and footrest) although measurements at the seat and backrest were obtained simultaneously. A comparison of the absorbed power at the different interfaces will not be accurate, but also not misleading, because the absorbed power at the feet was much smaller than at either the seat or the backrest (Figure 11).

\section{FIGURE 11 ABOUT HERE}

The overall power was calculated by summing the total absorbed power at the seat with the total absorbed power at the backrest. Statistical analysis showed no significant differences in the overall absorbed power between the four different postures, except between the feet hanging posture and the average thigh contact posture at both 0.625 and $1.25 \mathrm{~ms}^{-2}$ r.m.s. where there was greater absorbed power with the feet hanging posture $(p<0.05$, Wilcoxon).

\section{DISCUSSION}

The measurements show low absorbed power at low frequencies, consistent with the human body responding to vibration as an almost rigid system at low frequencies and consistent with previous measurements $[9,15,16]$.

The frequency of the first peak in the absorbed power at the seat without backrest (around $1 \mathrm{~Hz}$ ) is consistent with the principal resonance frequency evident in the foreand-aft apparent mass without a backrest [6] and in the absorbed power measured on a seat without a backrest $[15,16]$. The peak at $1 \mathrm{~Hz}$ has been explained in apparent mass studies as a result of body sway during fore-and-aft whole-body vibration. The decrease in the absorbed power at the seat at $1 \mathrm{~Hz}$ when a backrest was used is consistent with this explanation: the backrest reduced the sway at $1 \mathrm{~Hz}$ and hence decreased the force resulting from the sway and the absorbed power. A second peak in the absorbed power was evident between 3 and $4 \mathrm{~Hz}$ and was more pronounced when the feet were supported. With the feet hanging, the pendulum-like motion of the lower legs during vibration might have been out-of-phase with the motion of the body such that the total force on the seat was less than with the feet supported so that the lower legs moved in-phase with the upper body. A second peak between 3 and $4 \mathrm{~Hz}$ is also evident in the absorbed power on the seat reported by Rakheja et al. [16]. The absorbed power figures reported by Lundström and Holmlund [15] do not show this second peak, possibly due to the different conditions: they used sinusoidal vibration and a stationary footrest as opposed to random vibration and moving footrest as used in this study. 
The greater total absorbed power when the feet were hanging than when the feet were supported could be attributed to the greater mass of the body on the rigid seat in the feet hanging posture. Statistical analysis showed significant positive correlations between subject mass and the total power absorbed at the seat at all vibration magnitudes ( $p<0.004$, Spearman). This is consistent with increased absorbed power with increased body weight reported previously [21]. However, the observed increase in the total absorbed power with the minimum thigh contact posture compared with the other feet supported postures might be due to increased forces at the seat surface in the fore-and-aft direction as a result of rocking and bending modes of the body during fore-and-aft vibration. In the minimum thigh contact posture the body was resting on a small area (ischial tuberosities) making it easy to have a rocking mode. The significantly greater absorbed power at the backrest with the minimum thigh contact posture than with the other postures supports this explanation: rocking and bending modes of the body affect forces at the backrest as well as forces at the seat.

The absorbed power measured at the seat was found to have two peaks at similar frequencies whether or not the subjects used a backrest. However, the backrest reduced the absorbed power at the seat at low frequencies, including the first peak, while increasing the absorbed power at high frequencies, including the second peak. A similar effect of the backrest was seen in the absorbed power measured at the feet. This effect of backrests has also been seen in the apparent masses of seated subjects exposed to fore-and-aft vibration [20]. The reduction in the absorbed power at low frequencies may be due to stiffening of the body when in contact with a backrest. The reduction in absorbed power at the seat at low frequencies when using a backrest might also partly be explained by power absorbed at the backrest. The backrest was so beneficial at low frequencies that the absorbed power at the seat without a backrest was greater than the overall of the absorbed power at the seat and the backrest even though the magnitude of vibration between 0.25 and $10 \mathrm{~Hz}$ with the backrest was about 1.4 times greater than without the backrest: in both studies (i.e. without and with backrest), the same vibration magnitudes were used but over different frequency ranges $(0.25-20 \mathrm{~Hz}$ without backrest and $0.25-10 \mathrm{~Hz}$ with backrest).

The total absorbed power at the backrest was significantly greater than that at the seat surface, reflecting the high forces at the backrest. If discomfort (or injury) were assumed to be related to absorbed power, this provides further evidence of the importance of backrest vibration and suggests that vibration at this location should not be neglected when assessing vibration exposures (e.g. vibration dose values, VDV) or seat effective amplitude transmissibilities (SEAT) values [1]. 
Inter-subject variability in absorbed power may be attributed to differences in body mass, body build, stature, age, etc. There was greatest variability in absorbed power at the seat in the maximum thigh contact posture that may have arisen from differences in contact with the footrest. This posture was achieved by adjusting the height of the footrest until subjects said they felt their heels were just touching the footrest, so some may have been able to exert more force on the footrest than others. Subjects with less contact at the feet may have adopted different postures or made more postural adjustments during vibration. Some of the inter-subject variability in absorbed power at the back could be due to the height of the backrest not being adjusted for differences in subjects stature, so the point of contact between the back and the force platform differed between short and tall subjects.

The acceleration spectra used in this study were approximately flat in the frequency range of interest. Moreover, the vibration was in the fore-and-aft direction at all inputs (i.e. at the seat, back and feet) with no phase differences between inputs. In real situations, people are exposed to vibration with varying spectra, with multi-directional inputs, and with phase differences between these inputs. The spectra of absorbed power presented in this paper only apply to the conditions investigated, but they may be used to infer how the power absorbed in the human body is likely to depend on the frequency and the magnitude of vibration, and contact with footrests and backrests, in real vibration environments. The power absorbed at the seat, the back and the feet, and how these absorbed powers depend on the seat and body posture, may also help to explain and predict the effect of such factors on vibration discomfort.

\section{CONCLUSIONS}

During whole-body fore-and-aft vibration excitation, the power absorbed by the body at the seat shows two peaks: around $1 \mathrm{~Hz}$ and between 3 and $4 \mathrm{~Hz}$. The frequencies and magnitudes of the peak in absorbed power, as well as the total absorbed power, are affected by contact with footrests and backrests. The results suggest that backrests may be beneficial with low frequency fore-and-aft vibration but detrimental with high frequency fore-and-aft vibration. This is consistent with reported effects of backrests on both the apparent mass and the discomfort of seated subjects: backrests reduce the forces in the body at low frequencies but increase the forces at high frequencies. 


\section{REFERENCES}

[1] M.J.Griffin, Handbook of Human Vibration, London: Academic Press Limited, 1990.

[2] British Standards Institution, British Standard guide to measurement and evaluation of human exposure to whole-body vibration and repeated shock. British Standard BS 6841 (1987).

[3] International Organization for Standardization, Vibration and shock - evaluation of human exposure to whole-body vibration. ISO 2631-1 (1997).

[4] G.S. Paddan, M.J. Griffin, The transmission of translational seat vibration to the head. I. Vertical seat vibration, Journal of Biomechanics 21 (1988) 191-197.

[5] S. Rakheja, I. Stiharu, P.E. Boileau, Seated occupant apparent mass characteristics under automotive postures and vertical vibration, Journal of Sound and Vibration 253 (2002) 57-75.

[6] N. Nawayseh, M.J. Griffin, Non-linear dual-axis biodynamic response to fore-and-aft whole-body vibration, Journal of Sound and Vibration 282 (2005) 831-862.

[7] N. Nawayseh, M.J. Griffin, Non-linear dual-axis biodynamic response to vertical whole-body vibration, Journal of Sound and Vibration 268 (2003) 503-523.

[8] E.B. Weis, N.P. Clarke, J.W. Brinkley, P.J. Martin, Mechanical impedance as a tool in research on human response to acceleration, Aerospace Medicine 35 (1964) 945950.

[9] R.A. Lee, F. Pradko, Analytical analysis of human vibration, Automotive Engineering Congress, Detroit, Mich., U.S.A, January 1968.

[10] N. Nawayseh, M.J. Griffin, Power absorbed during whole-body vertical vibration: Effects of sitting posture, backrest, and footrest, Journal of Sound and Vibration 329 (2010) 2928-2938.

[11] N.J. Mansfield, M.J. Griffin, Effect of magnitude of vertical whole-body vibration on absorbed power for the seated human body, Journal of Sound and Vibration 215 (1998) 813-825.

[12] R. Lundström, P. Holmlund, L. Lindberg, Absorption of energy during vertical whole-body vibration exposure, Journal of Biomechanics 31 (1998) 317-326.

[13] P. Holmlund, R. Lundström, Mechanical impedance of the human body in the horizontal direction. Journal of Sound and Vibration 215 (1998) 801-812.

[14] G.S. Paddan, M.J. Griffin, The transmission of translational seat vibration to the head. II. Horizontal seat vibration. Journal of Biomechanics 21 (1988) 199-206.

[15] R. Lundström, P. Holmlund, Absorption of energy during whole-body vibration exposure, Journal of Sound and Vibration 215 (1998) 789-799.

[16] S. Rakheja, S. Mandapuram, R.G. Dong, Energy absorption of seated occupants exposed to horizontal vibration and role of back support condition, Industrial Health 46 (2008) 550-566.

[17] K.C. Parsons, M.J. Griffin and E.M. Whitham, Vibration and comfort: III. Translational vibration of the feet and back, Ergonomics 25 (1982) 705-719.

[18] I.H. Wyllie and M.J. Griffin, Discomfort from sinusoidal oscillation in the pitch and fore-and-aft axes at frequencies between 0.2 and $1.6 \mathrm{~Hz}$, Journal of Sound and Vibration 324 (2009) 453-467. 
[19] M. Morioka and M.J. Griffin, Frequency Weightings for Fore-and-aft Vibration at the Back: Effect of Contact Location, Contact Area, and Body Posture, Industrial Health 48 (2010) 538-549.

[20] N. Nawayseh, M.J. Griffin, Tri-axial forces at the seat and backrest during wholebody fore-and-aft vibration, Journal of Sound and Vibration 281 (2005) 921-942.

[21] R. Lundström, P. Holmlund, L. Lindberg, Absorption of energy during vertical whole-body vibration exposure, Journal of Biomechanics 31 (1998) 317-326. 


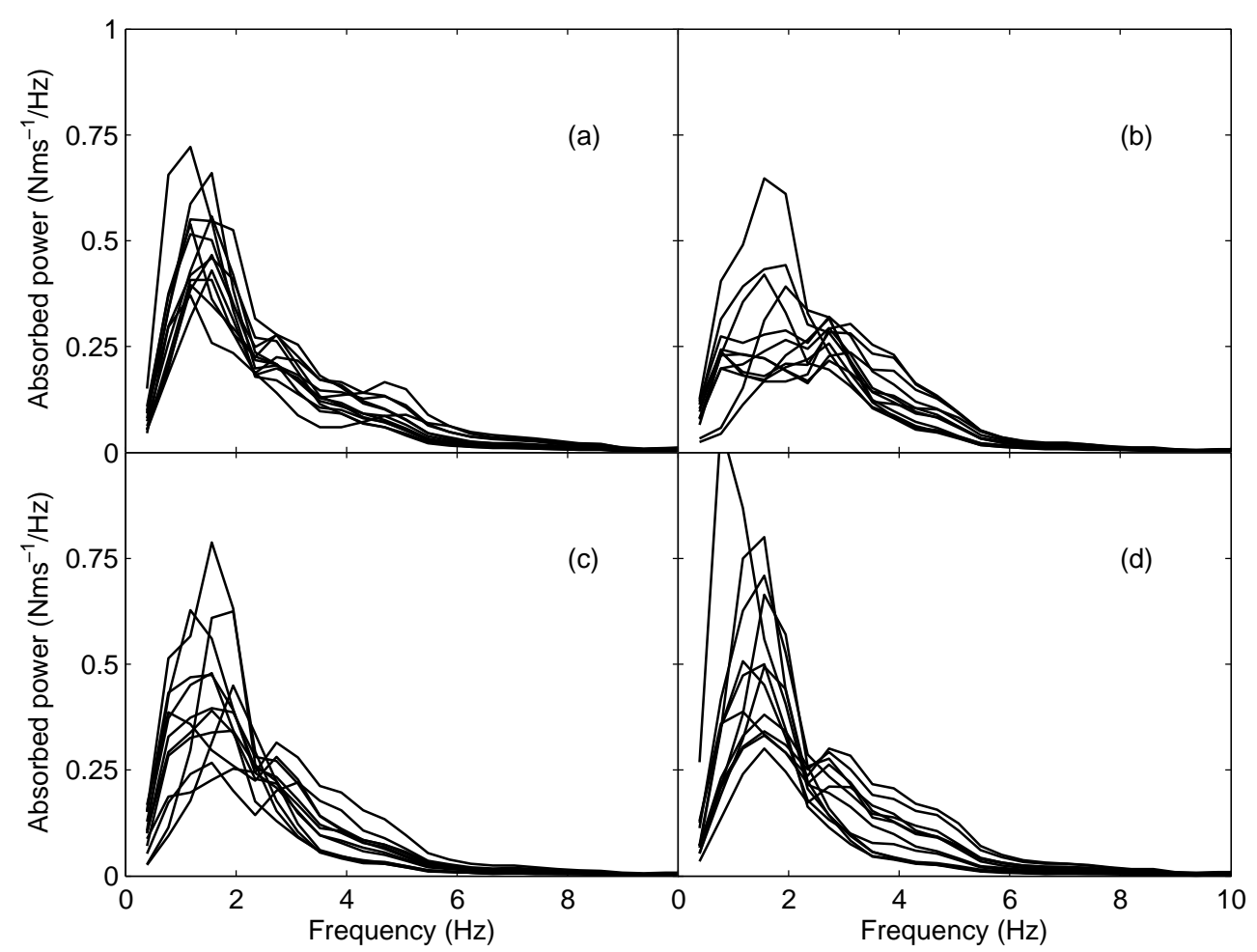

Figure 1 Absorbed power measured at the seat without backrest at $1.25 \mathrm{~ms}^{-2}$ r.m.s. with four sitting postures: (a) feet hanging; (b) maximum thigh contact; (c) average thigh contact; and (d) minimum thigh contact (linear scales, individual data of 12 subjects). 

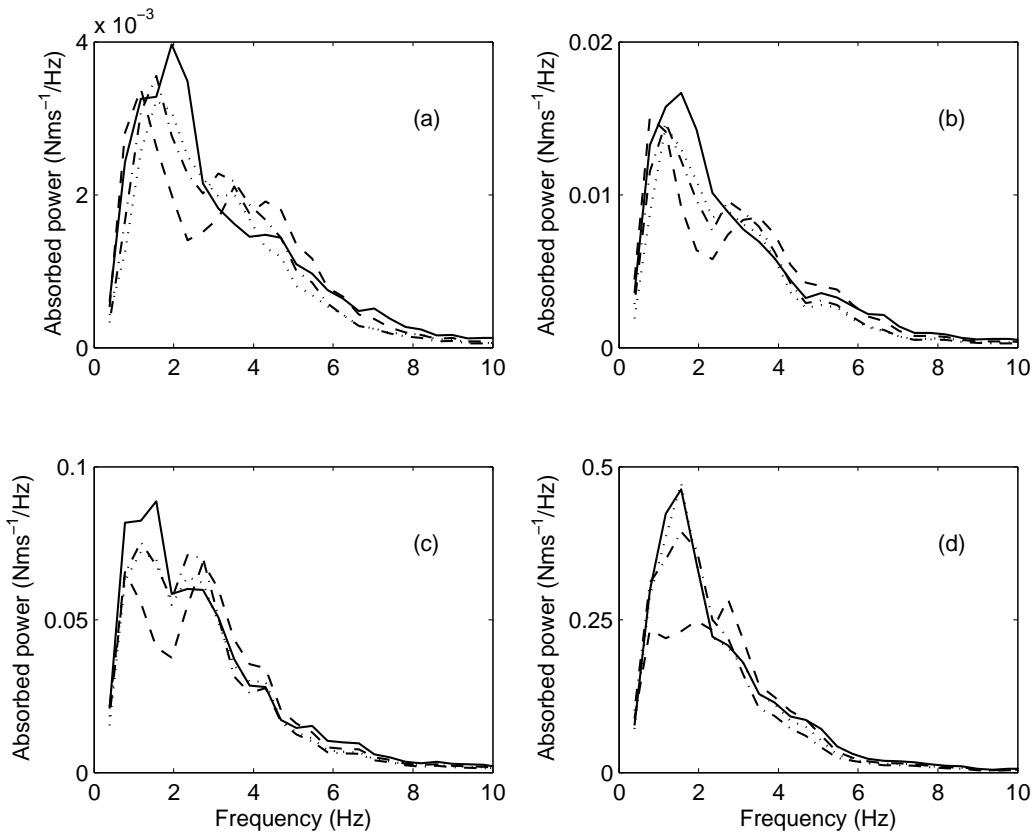

Figure 2 Effect of posture on absorbed power at the seat without backrest at four vibration magnitudes: (a), $0.125 \mathrm{~ms}^{-2}$ r.m.s.; (b), $0.25 \mathrm{~ms}^{-2}$ r.m.s.; (c), $0.625 \mathrm{~ms}^{-2}$ r.m.s.; (d), $1.25 \mathrm{~ms}^{-2}$ r.m.s. ——, feet hanging; - - - - maximum thigh contact; —-, average thigh contact; ; minimum thigh contact (linear scales; medians of 12 subjects). 


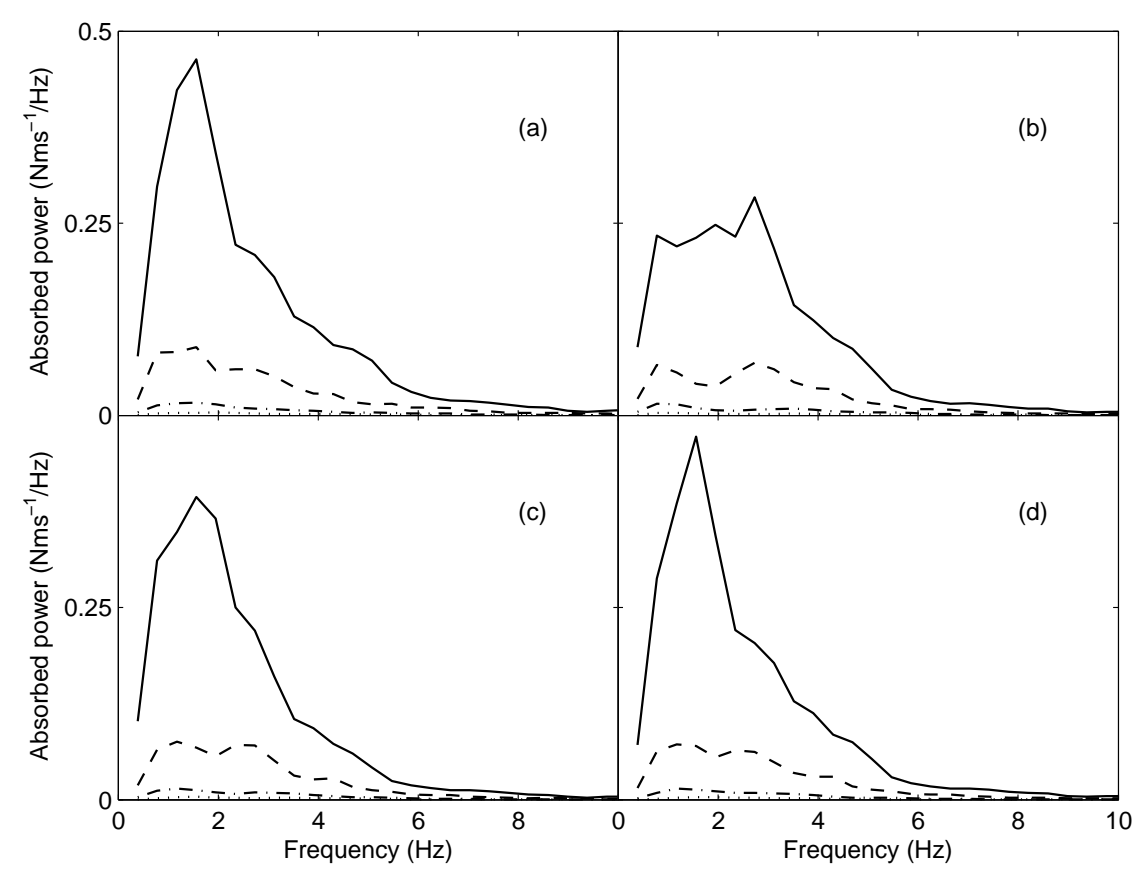

Figure 3 Effect of vibration magnitude on absorbed power at the seat with four sitting postures: (a), feet hanging; (b), maximum thigh contact; (c), average thigh contact; (d), minimum thigh contact. ; $0.125 \mathrm{~ms}^{-2}$ r.m.s.; - $0.25 \mathrm{~ms}^{-2}$ r.m.s.;,$---- 0.625 \mathrm{~ms}^{-2}$ r.m.s.; —_ $1.25 \mathrm{~ms}^{-2}$ r.m.s. (linear scales; medians of 12 subjects). 


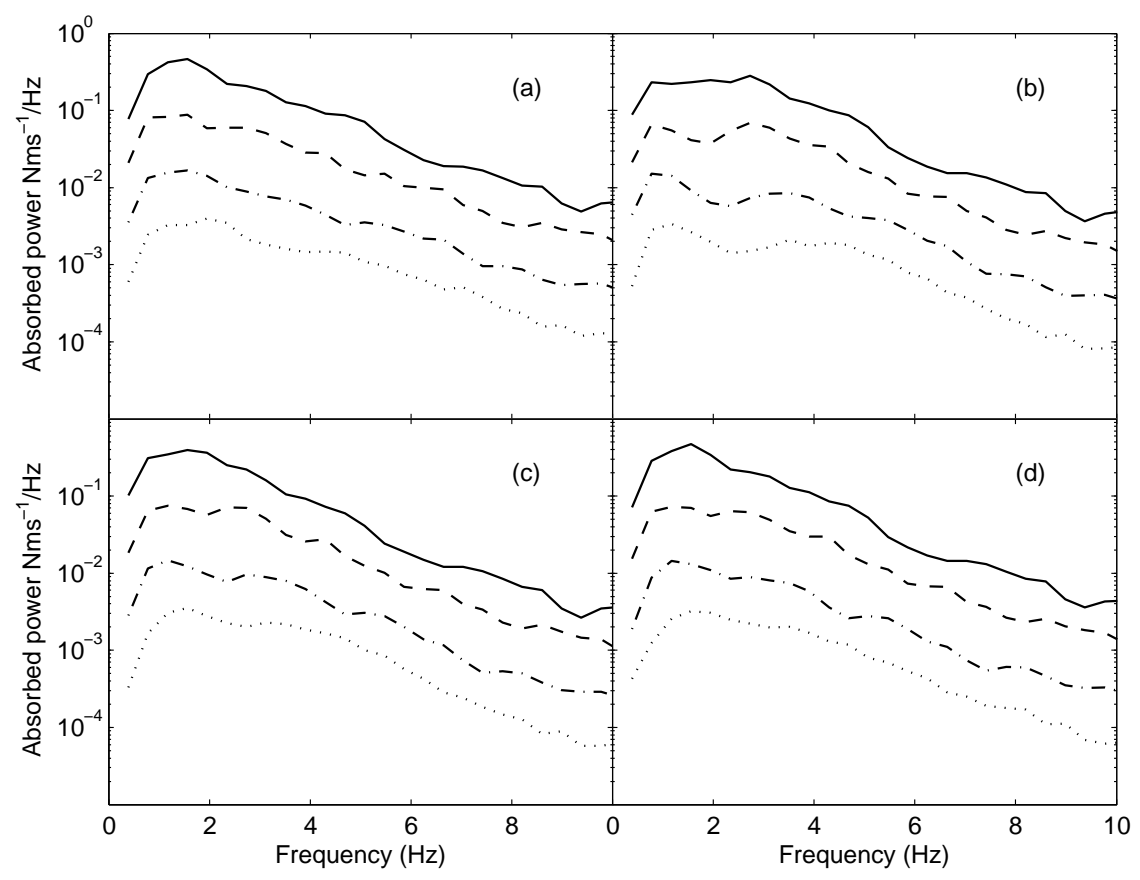

Figure 4 Effect of vibration magnitude on absorbed power at the seat with four sitting postures: (a), feet hanging; (b), maximum thigh contact; (c), average thigh contact; (d), minimum thigh contact. ; $0.125 \mathrm{~ms}^{-2}$ r.m.s.; - $0.25 \mathrm{~ms}^{-2}$ r.m.s.;,$---- 0.625 \mathrm{~ms}^{-2}$ r.m.s.; —— $1.25 \mathrm{~ms}^{-2}$ r.m.s. (logarithmic scales; medians of 12 subjects). 

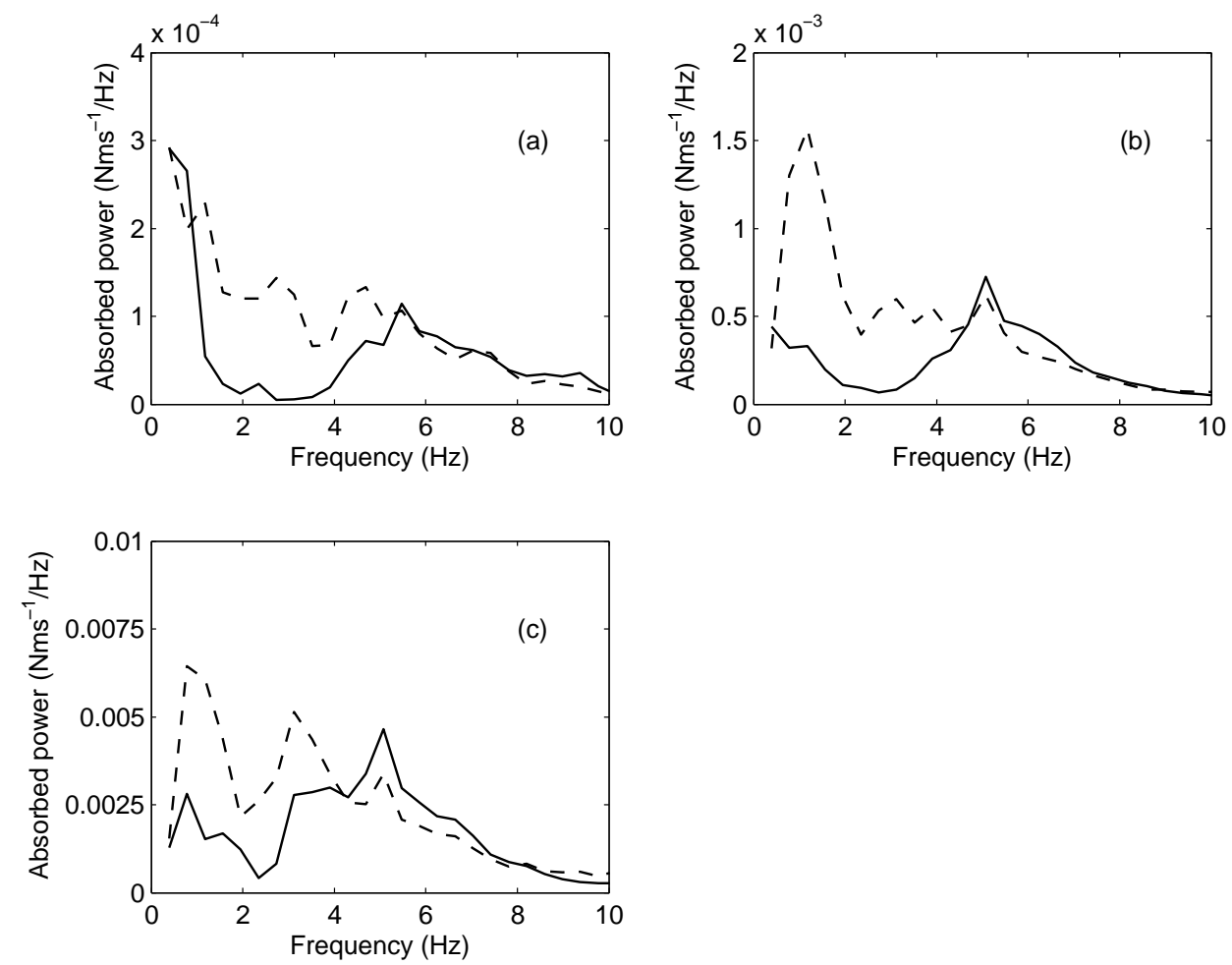

Figure 5 Absorbed power measured at the feet with the average thigh contact posture: (a), $0.125 \mathrm{~ms}^{-2}$ r.m.s.; (b), $0.25 \mathrm{~ms}^{-2}$ r.m.s.; (c), $0.625 \mathrm{~ms}^{-2}$ r.m.s. - - - -, without backrest; , with backrest (linear scales, individual data of 6 subjects). 

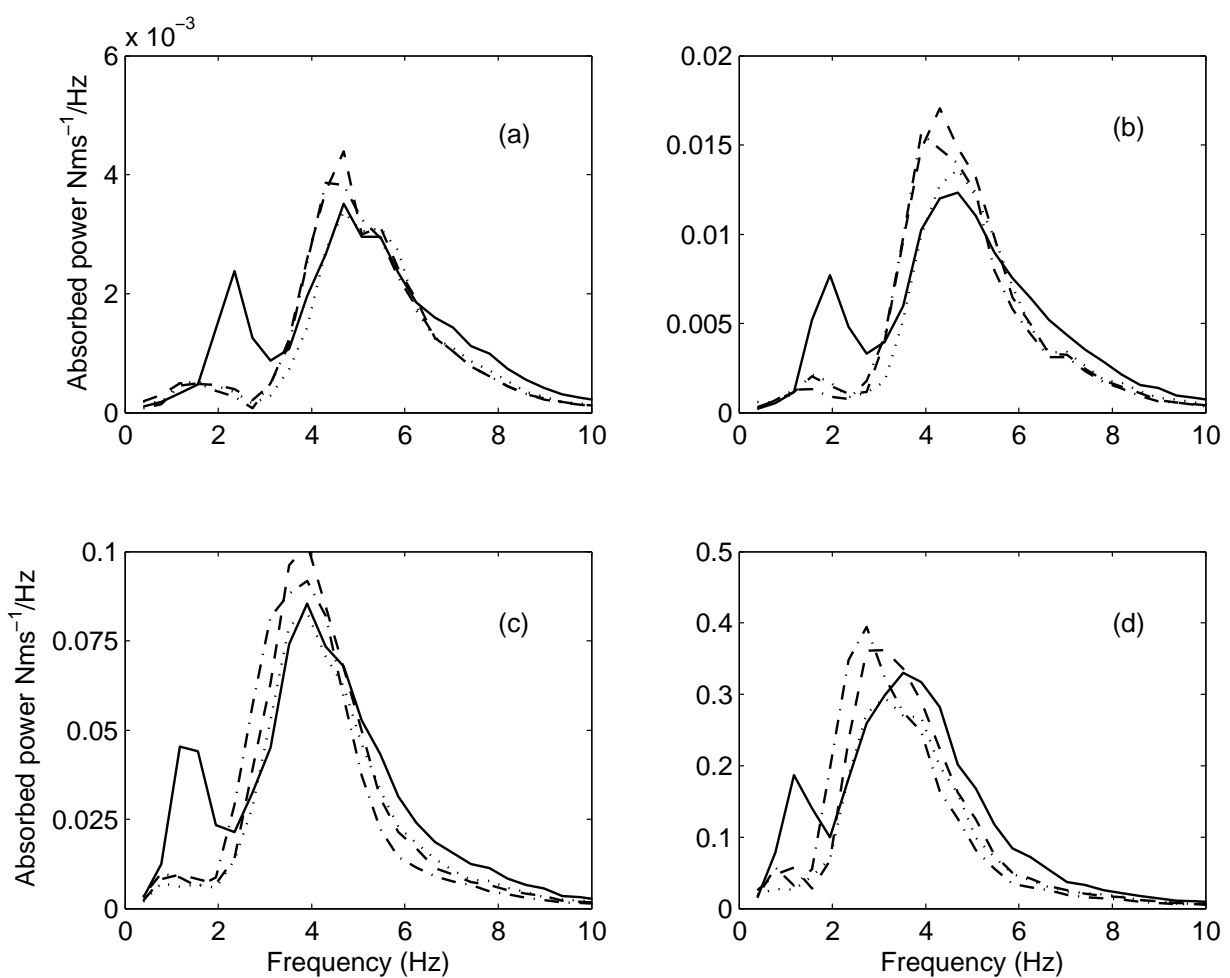

Figure 6 Effect of posture on absorbed power at the seat with backrest at four vibration magnitudes: (a), $0.125 \mathrm{~ms}^{-2}$ r.m.s.; (b), $0.25 \mathrm{~ms}^{-2}$ r.m.s.; (c), $0.625 \mathrm{~ms}^{-2}$ r.m.s.; (d), 1.25 $\mathrm{ms}^{-2}$ r.m.s. _- feet hanging; - - - - maximum thigh contact; ___, average thigh contact; ; minimum thigh contact (linear scales; medians of 12 subjects). 


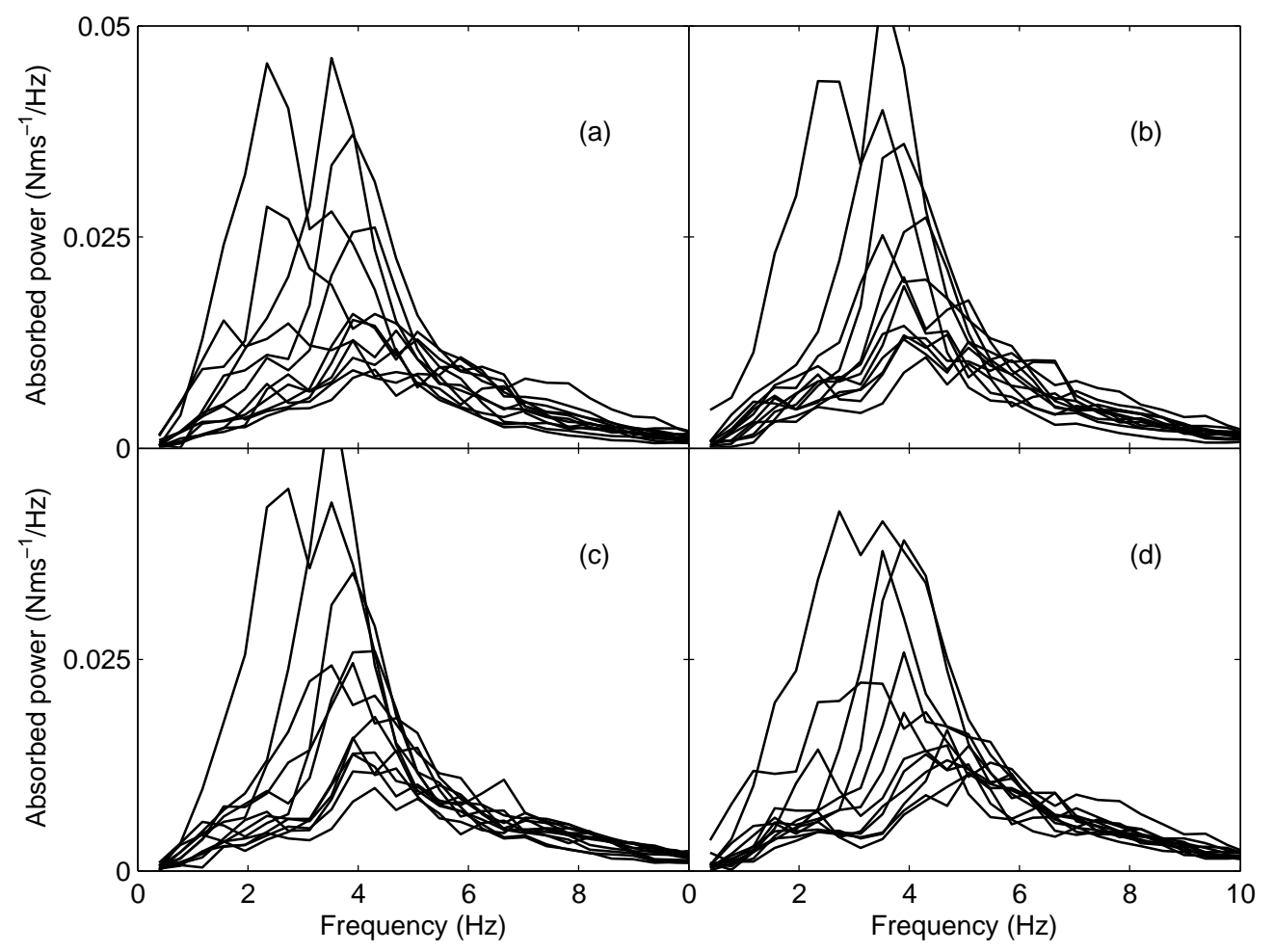

Figure 7 Absorbed power measured at the backrest at $0.25 \mathrm{~ms}^{-2}$ r.m.s. with four sitting postures: (a) feet hanging; (b) maximum thigh contact; (c) average thigh contact; and (d) minimum thigh contact (linear scales, individual data of 12 subjects). 

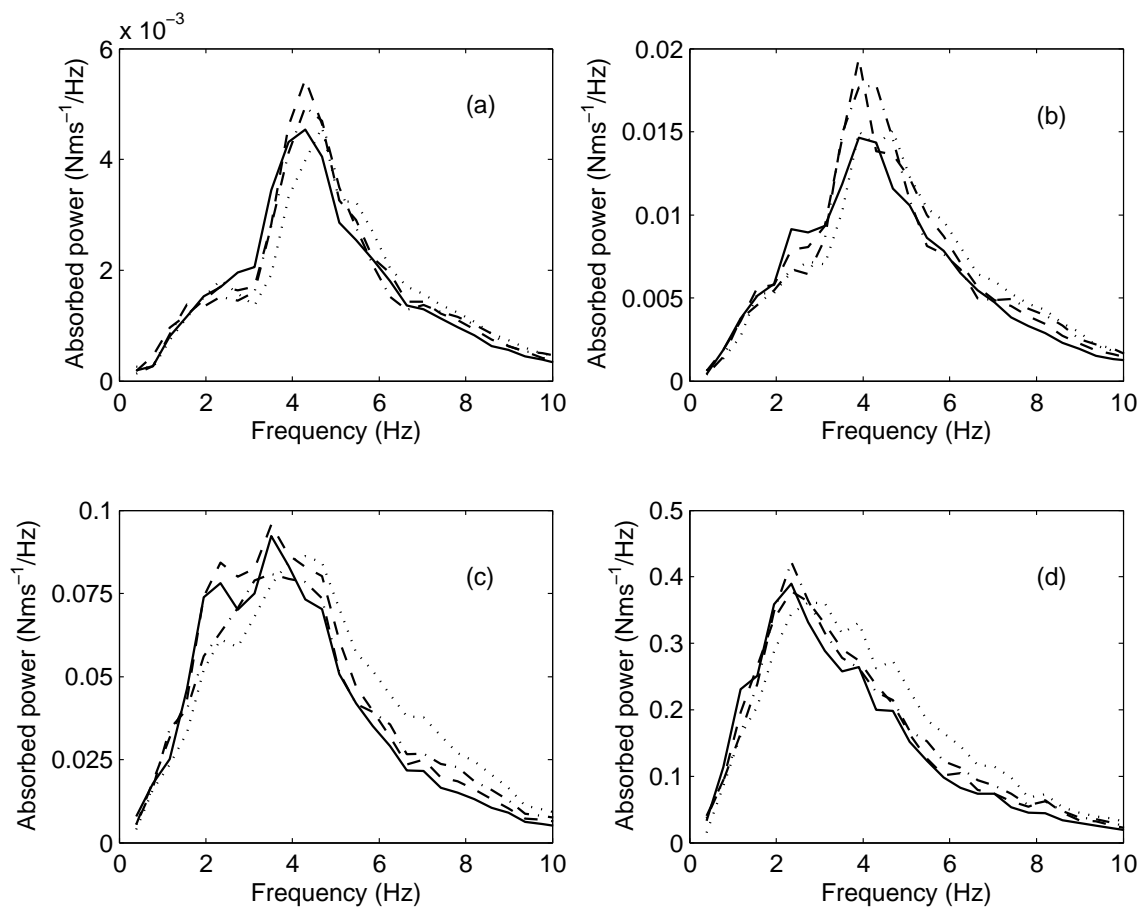

Figure 8 Effect of posture on absorbed power at the backrest at four vibration magnitudes: (a), $0.125 \mathrm{~ms}^{-2}$ r.m.s.; (b), $0.25 \mathrm{~ms}^{-2}$ r.m.s.; (c), $0.625 \mathrm{~ms}^{-2}$ r.m.s.; (d), 1.25 $\mathrm{ms}^{-2}$ r.m.s. ——, feet hanging; - - - maximum thigh contact; - _ , average thigh contact; ; minimum thigh contact (linear scales; medians of 12 subjects). 


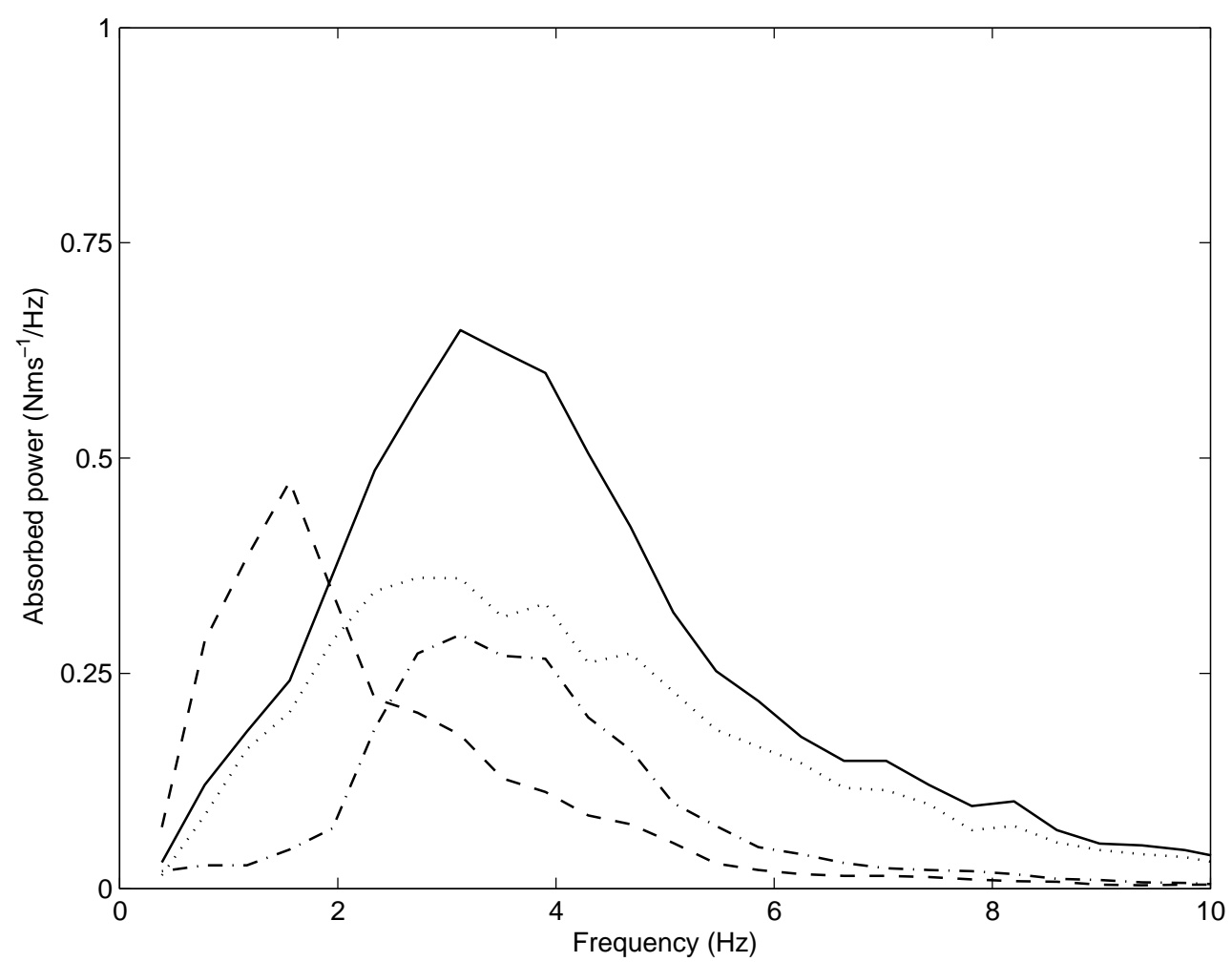

Figure 9 Absorbed power at the seat and backrest in the minimum thigh contact posture with $1.25 \mathrm{~ms}^{-2}$ r.m.s. fore-and-aft vibration: $--_{-}$, at the seat with no backrest; —- at the seat with backrest; ; at the backrest; —_, sum of absorbed power at the seat and the backrest (medians of 12 subjects). 

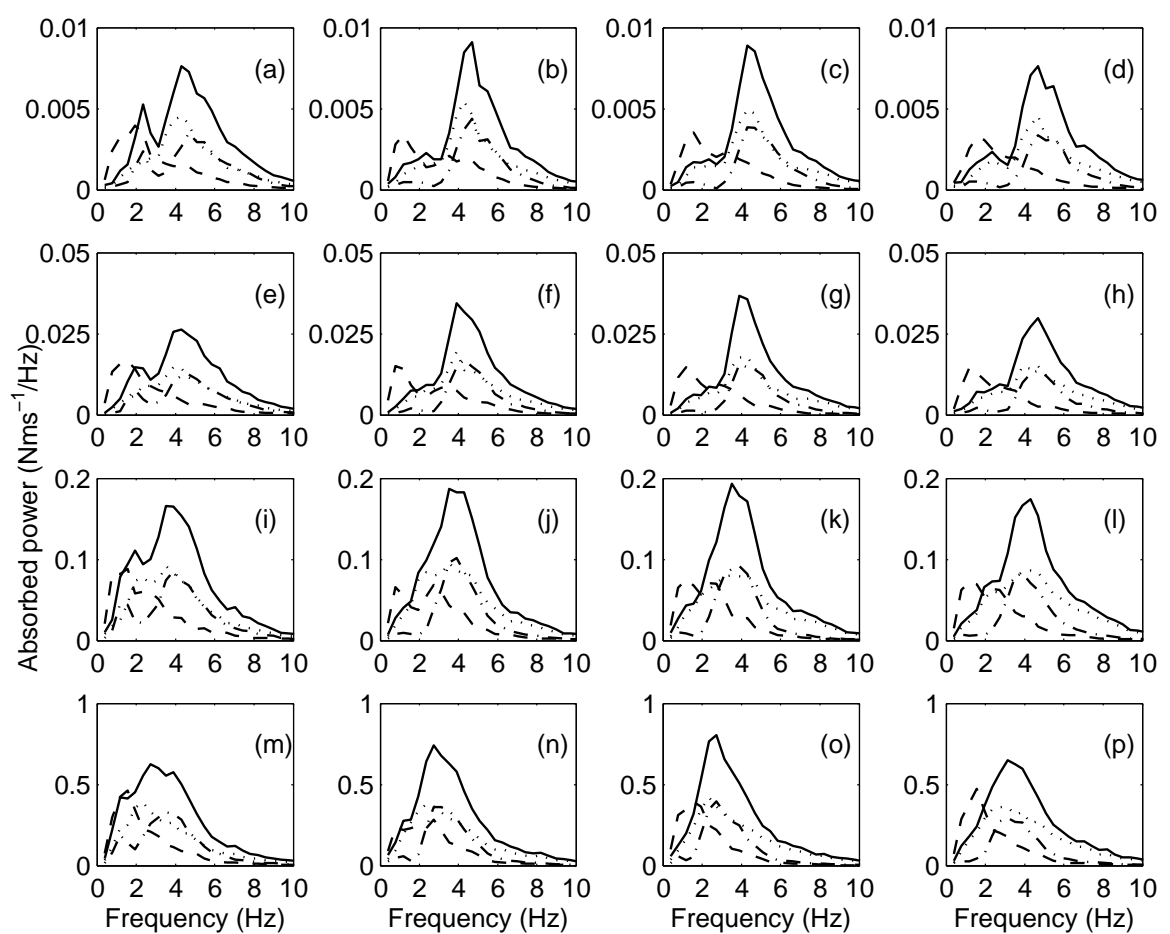

Figure 10 Absorbed power at the seat and backrest: - - - -, at the seat with no backrest; - - - at the seat with backrest; ; at the backrest; - - , overall at the seat and backrest. (a), (b), (c), (d), $0.125 \mathrm{~ms}^{-2}$ r.m.s.; (e), (f), (g), (h), $0.25 \mathrm{~ms}^{-2}$ r.m.s.; (i), (j), (k), (l), $0.625 \mathrm{~ms}^{-2}$ r.m.s.; (a), (b), (c), (d), $1.25 \mathrm{~ms}^{-2}$ r.m.s. (a), (e), (i), (m), feet hanging; (b), (f), (j), (n), maximum thigh contact; (c), (g), (k), (o), average thigh contact; (d), (h), (l), (p), minimum thigh contact; (linear scales; medians of 12 subjects). 


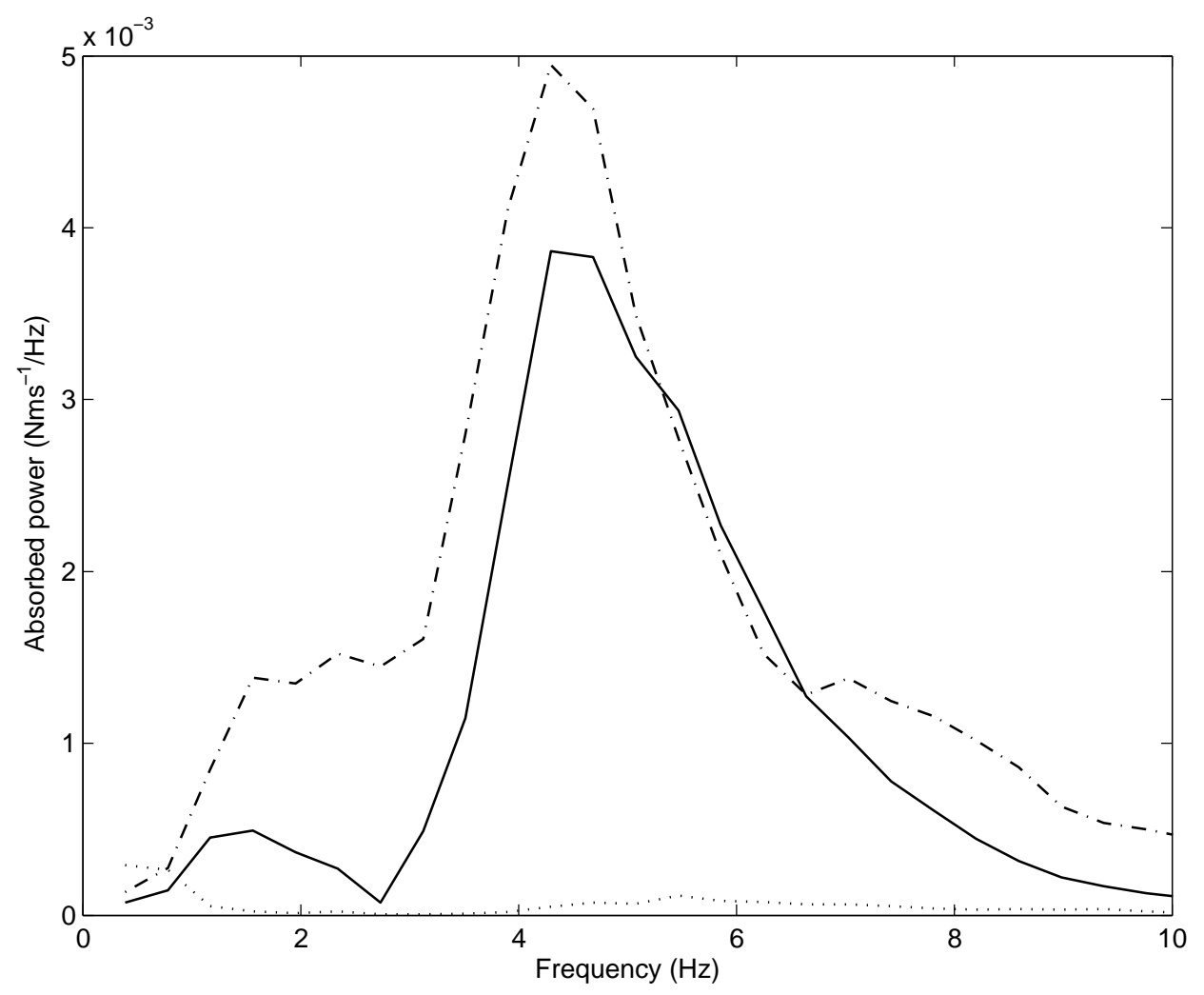

Figure 11 Median absorbed power measured at the seat, at the backrest, and at the footrest with the average thigh contact posture at $0.625 \mathrm{~ms}^{-2}$ r.m.s.: — - , at the seat; - - 